\title{
P04-1-7 Poster session
}

\section{Drebrin regulates sub-spine localization of Calcium/calmodulin-dependent kinase II $\beta$}

\author{
Hiroyuki Yamazaki, Tomoaki Shirao
}

Neurobiology and Behavior, Gunma University Graduate School of Medicine, Japan

Dendritic spines are actin-rich small protrusions that contain postsynaptic components of excitatory synapse. Many actinbinding proteins have been identified as spine-resident protein, and they regulate actin-cytoskeleton through diverse processes. Drebrin is a major F-actin binding protein in neurons, and is localized in the center of dendritic spines. In this study, we isolated CaMKII $\beta$ as a drebrin-binding protein by yeast two-hybrid screen and investigated drebrin-CaMKII $\beta$ relationship in dendritic spines. CaMKII $\beta$ is localized in dendritic spines more than in dendritic shaft. However, drebrin knockdown (KD) caused diffuse localization of CaMKII $\beta$ in dendrites, suggesting that drebrin anchors CaMKII $\beta$ in dendritic spines. To analyze drebrin-dependence of CaMKII $\beta$ stability in dendritic spine more directly, we performed fluorescence recovery after photobleaching (FRAP) experiments on individual dendritic spines. We found that the stable fraction of CaMKII $\beta$ in drebrin-KD neurons was greater than that of control neurons. This result seems to be inconsistent with the results that CaMKII $\beta$ diffused in dendrites of drebrin-KD neurons. We consequently think that drebrin-independent stable pool became dominant in drebrin-KD neurons. To test the hypothesis, we used stochastic optical reconstruction microscopy (STORM) to elucidate the localization of drebrin and CaMKII $\beta$ in a dendritic spine on the nanometer scale. Our super resolution data showed that CaMKII $\beta$ is partially co-localize with drebrin in the inner side of dendritic spines. In addition, the co-localization of CaMKII $\beta$ and drebrin was separated by NMDA receptor activation, suggesting that active form of CaMKII $\beta$ is free from drebrin. Furthermore, FRAP analysis showed that NMDA receptor activation markedly increased the stable fraction of CaMKII $\beta$. Together, these results suggest that CaMKII $\beta$ is localized in dendritic spines as both drebrin-dependent pool and drebrin-independent more stable pool. 